\title{
Artigo original \\ Força de membros inferiores como indicador de incapacidade funcional em idosos
}

\author{
Rafaela G. dos Santos \\ Sheilla Tribess \\ Joilson Meneguci \\ Letícia L. A. da G. Bastos \\ Renata Damião \\ Jair S. Virtuoso Júnior \\ Universidade Federal do Triângulo Mineiro
}

\begin{abstract}
Resumo - O propósito deste estudo foi discriminar a quantidade de repetições no teste sentar e levantar da cadeira na estimativa da incapacidade funcional. Estudo epidemiológico de corte transversal com amostra probabilística de 622 indivíduos, idade $\geq 60$ anos. A capacidade funcional autopercebida foi analisada como variável dicotômica: ausência de dependência versus dependência na realização de atividade básicas (ABVD) e instrumentais da vida diária (AIVD). Aplicou-se o teste sentar e levantar da cadeira durante 30 segundos (número de repetições máximas) para avaliar a força de membros inferiores. O teste mostrou-se como discriminador da incapacidade funcional devido os valores apresentados pelas áreas sob as curvas ROC serem $>0,60$. O ponto de corte para determinar a incapacidade funcional nas ABVD foi $\leq 10$ repetições (homens) $\mathrm{e} \leq 9$ repetições (mulheres); nas AIVD $\leq 14$ repetições (homens) $\mathrm{e} \leq 10$ repetições (mulheres). A força de membros inferiores pode ser utilizada como preditor da incapacidade funcional nas ABVD e AIVD.
\end{abstract}

Palavras-chaves: membros inferiores, força muscular, pessoa com incapacidade, idoso, curva ROC

Abstract- - "Lower limb strength as an indicator of functional disability in older individuals." The purpose of this study was to identify the number of repetitions of the sitting-rising test from a chair necessary to estimate a functional disability in older individuals. An epidemiological cross-sectional study was conducted with a random sample of 622 individuals, aged $\geq 60$ years. The functional capacity was investigated as a dichotomous variable: absence of dependency versus dependency for performing basic activities (BADL) and instrumental activities of daily living (IADL). We employed the sitting-rising test from a chair for 30 seconds (maximum repetitions) to assess the strength of the lower limbs. The test proved to be a good discriminator of functional disability due to the values $>0.60$ which were identified in the areas under the ROC curves. The cutoff point for determining disability in the BADL was: $\leq 10$ repetitions (men) and $\leq 9$ repetitions (women); in the IADL: $\leq 14$ repetitions (men) and $\leq 10$ repetitions (women). The strength of the lower limbs can be used as a predictor of functional disability in BADL and IADL.

Keywords: lower extremity, muscle strength, functional disabilities, aging, ROC curve

Resumen - "Fuerza de las extremidades inferiores como un indicador de la incapacidad funcional en ancianos." El propósito de este estudio fue discriminar la cantidad de repeticiones en la prueba de sentarse y levantarse de la silla en la estimación de la discapacidad funcional. Estudio epidemiológico transversal con una muestra aleatoria de 622 individuos con edad $\geq 60$ años. La capacidad funcional autopercibida se analizó como una variable dicotómica: la falta de dependencia frente a la dependencia para realizar las actividades básicas (ABVD) y actividades instrumentales de la vida diaria (AIVD). Se aplicó la prueba de sentarse y levantarse de una silla durante 30 segundos (repeticiones máximas) para evaluar la fuerza de los miembros inferiores. La prueba resultó ser discriminatorio por discapacidad las cifras correspondientes a las áreas bajo las curvas ROC fueron $>0,60$. El punto de corte para determinar la discapacidad en las ABVD fue $\leq 10$ repeticiones (hombres) y $\leq 9$ repeticiones (mujeres), el AIVD $\leq 14$ repeticiones (hombres) y $\leq 10$ repeticiones (mujeres). La fuerza de los miembros inferiores puede ser utilizada como un indicador de la incapacidad funcional en ABVD y AIVD.

Palabras claves: extremidad inferior, fuerza muscular, personas con discapacidad, anciano, curva ROC 


\section{Introdução}

No Brasil, nas últimas décadas, tem havido um aumento do contingente de pessoas com idade igual ou superior a 65 anos, com a passagem do representativo total da população de 4,8\% em 1991 para 5,9\% em 2000 e 7,4\% em 2010 segundo o Instituto Brasileiro de Geografia e Estatística [IBGE] (2010). $\mathrm{O}$ acelerado aumento da expectativa de vida tem gerado preocupações com as condições de saúde dos longevos (Ezzati, Friedman, Kulkarni, \& Murray, 2008). A Organização Mundial de Saúde [OMS] em suas estratégias chama atenção para que a população idosa passe seus anos de vida de forma saudável, com elevada e completa funcionalidade em cada fase da vida (Barreto et al., 2005).

$\mathrm{O}$ processo do envelhecimento que vem acompanhado com comportamentos inadequados a saúde, pode favorecer ao surgimento de patologias e o comprometimento funcional (Maciel, 2010).

A incapacidade funcional possui elevada prevalência na população idosa e remete a um quadro de dependência e de vulnerabilidade à morbidades e mortalidade (Arai \& Hirose, 2013). Os primeiros modelos teóricos explicativos para incapacidade funcional remetem para uma sequência precedida pela patologia, passando pela deficiência e limitações funcionais (Nagi, 1991; Verbrugge \& Jette, 1994). $\mathrm{Na}$ atualidade, o modelo de Função e Incapacidade Funcional (ICDH-2) incorpora o modelo biopsicossocial para abordar questões de comprometimento à saúde e serve de referência para Classificação Internacional de Funcionalidade (Worral, McCooey, Davidson, Larkins, \& Hickson, 2002).

A incapacidade funcional pode ser compreendida como limitações em desempenho de papéis socialmente definidos e tarefas dentro de um ambiente sociocultural (Alves, Leite, \& Machado, 2008).

A Sociedade Americana de Geriatria estratificou as atividades inerentes ao construto da capacidade funcional em: básicas (ABVD), intermediárias (AIVD) e avançadas (AAVD) (Reuben \& Solomon, 1989). As ABVD incluem as atividades de autocuidado, já as AIVD englobam as ABVD e incluem tarefas essenciais para a manutenção da independência na comunidade onde vive. As AAVD referemse às funções necessárias para se viver sozinho, incluem a manutenção das funções ocupacionais, recreacionais e prestação de serviços comunitários (Virtuoso, Mendes \& Tribess, 2010). A execução de forma segura e independente de tais ABVD e AIVD envolvem aspectos essenciais como a força muscular (Chien, Kuo, \& Wu, 2010). A perda da força muscular com o avançar da idade (dinapenia) é parcialmente explicada pela diminuição na massa muscular e a sarcopenia (Manini \& Clark, 2010).

Além da força muscular, a flexibilidade, a resistência, a agilidade e o equilíbrio dinâmico também são necessários (Jones, Rikli, \& Beam, 1999) visto que são responsáveis por uma transição bem sucedida na atividade de sentar e levantar, sendo esta fundamental para a independência (Roorda, Roebroeck, Lankhorst, Tilburg, \& Bouter, 1996). Estes aspectos podem ser quantificados por meio de testes de desempenho e relacionados à incapacidade funcional
(Rikli \& Jones, 2013).

Apesar das evidências da relação da força muscular de membros inferiores com a condição funcional (McGough, Logsdon, Kelly, \& Teri, 2013; Wennie, Perera, Swearingen, \& Studenski, 2010), há lacuna na literatura quanto os estudos que possam analisar o potencial preditivo e os possíveis escores dos testes de desempenho como discriminadores da incapacidade funcional.

O desempenho no teste relativamente simples de sentar e levantar da cadeira pode ser indicador útil na prevenção da incapacidade em procedimentos de triagem das condições de saúde da população idosa. O conhecimento dos escores discriminadores da incapacidade funcional favorece aos gestores de saúde pública a tomar decisões em relação às intervenções necessárias a integridade do estado funcional da pessoa idosa.

Com base nessas considerações, o objetivo do presente estudo foi analisar o potencial preditivo e estimar os pontos de corte da força de membros inferiores para incapacidade funcional nas ABVD e AIVD, em pessoas idosas.

\section{Método}

\section{Desenho do estudo}

Estudo observacional, analítico de corte transversal, parte integrante do projeto "Estudo Populacional de Atividade Física e Envelhecimento (EPAFE)", o qual teve como referência uma população de 10.683 pessoas, com 60 anos ou mais, de ambos os sexos, residente na zona urbana do município de Uberaba, MG.

\section{Caracterização da amostra}

Participaram do estudo 622 idosos, sendo o tamanho da amostra definido de acordo com os procedimentos propostos por Luiz e Magnani (2000) para populações finitas. Para o cálculo da amostra adotou-se nível de significância de 5\% (correspondendo a um intervalo de confiança de 95\%, z [a]/ $2=1,96$ ) e erro tolerável de amostragem de $3 \%$. Este primeiro cálculo resultou em amostra mínima de 490 idosos, porém tendo o intuito de explorar associações ajustadas entre os componentes analisados, a amostra foi aumentada em $20 \%$, resultando assim uma amostra mínima de 588 idosos e posteriormente acrescentou-se mais $10 \%$ para eventuais perdas, totalizando 647.

Os idosos foram selecionados de forma aleatória, utilizando o software Epi Info, considerando a coleta de dados em base domiciliar, e os sujeitos do estudo representativos a zona urbana do município, do tipo proporcional ao distrito, equipe de saúde da família e sexo. Os critérios de inclusão dos idosos foram não possuir dificuldades na acuidade visual e auditiva em grau severo, não fazer uso de cadeiras de rodas, não estar acamados provisória ou definitivamente, não possuir sequelas graves de acidente vascular encefálico (AVE) com perda localizada de força e não possuir doença em estágio terminal. 


\section{Procedimentos éticos}

Esta pesquisa seguiu os princípios éticos presentes na Resolução nº196/96, do Conselho Nacional de Saúde. Os protocolos de pesquisa foram avaliados e aprovados pelo Comitê de Ética em Pesquisa com Seres Humanos da Universidade Federal do Triângulo Mineiro - UFTM (Parecer $\left.n^{\circ} 1521 / 2009\right)$.

\section{Variáveis mensuradas}

Os idosos que aceitaram participar do estudo assinaram termo de livre consentimento e responderam um questionário estruturado com informações sociodemográficas e de saúde, em forma de entrevista individual, e foram submetidos a avaliação antropométrica e desempenho físico de força de membros inferiores.

Os dados sociodemográficos foram idade; escolaridade (anos de estudo); estado civil (solteiro casado/vivendo com parceiro, viúvo, divorciado/separado); arranjo familiar (mora só, cônjuge ou outro, mora com filhos, mora com netos) e renda familiar (quantidade de salários-mínimos).

Em relação aos indicadores de saúde foram avaliadas a percepção do estado de saúde (positiva, negativa); déficit cognitivo (ausência, presença), sendo avaliado pelo MiniExame do Estado Mental (Folstein, Folstein, \& McHugh, 1975), adotando o ponto de corte proposto por Almeida (1998); presença de doenças referidas; consumo de medicamentos; ocorrência de quedas; hospitalização e capacidade funcional investigada como uma variável dicotômica: ausência de dependência versus dependência para a realização das ABVD e AIVD.

A incapacidade funcional para realização das ABVD foi determinada pelo índice de Katz, que contempla atividades de autocuidado, como, tomar banho, vestir-se, ir ao banheiro, deitar e levantar da cama, comer e controle das funções de urinar e/ou evacuar (Lino, Pereira, Camacho, Ribeiro, \& Buksman, 2008). A incapacidade funcional para realização de AIVD foi avaliada pela escala de Lawton adaptada ao contexto brasileiro, que relaciona atividades de telefonar, utilização de meio de transporte para deslocamento a lugares distantes, realização de compras, preparo da própria refeição, limpeza e arrumação da casa, controle do consumo de medicamentos e com a administração das finanças próprias (Santos \& Virtuoso, 2008).

$\mathrm{Na}$ avaliação antropométrica foram mensuradas a massa corporal, com o auxílio de uma balança digital de marca Filizola, e estatura, por um estadiômetro, adotando-se as padronizações internacionais de antropometria (Lohman, Roche, \& Martorell, 1998). O desempenho físico de força de membros inferiores foi avaliado pelo número máximo de repetições do teste de sentar e levantar da cadeira em 30 segundos de acordo com a bateria de testes físicos de Fullerton (Rikli \& Jones, 2008).

\section{Procedimento estatístico}

Para análise dos dados, confeccionou-se um banco de dados, no software Epidata, versão 3.1b. Após verificação da normalidade dos dados pelo teste de KolmogorovSmirnov, aplicou-se o teste $t$ de Student para amostras independentes (dados contínuos) e o teste qui-quadrado (dados categóricos) para comparação e distribuição das variáveis segundo ausência de dependência versus dependência para a realização de ABVD e AIVD. Os testes foram realizados no pacote estatístico SPSS 20 (Statistical Package for the Social Sciences - SPSS), adotou-se a significância de $5 \%$.

O poder preditivo e os pontos de corte do número de repetições máximas no teste de sentar e levantar, separados por sexo, para incapacidade funcional foram identificados por meio das curvas Receiver Operating Characteristic (ROC); analisados através do programa estatístico MedCal versão11.4.4.

Inicialmente, foi identificada a área total sob a curva ROC entre o número de repetições no teste de sentar e levantar e a incapacidade funcional na realização de ABVD e AIVD. Quanto maior a área sob a curva ROC, maior o poder discriminatório do teste de sentar e levantar para incapacidade funcional. Utilizou-se intervalo de confiança (IC) a $95 \%$, Em um segundo momento, a sensibilidade e a especificidade foram calculadas no estabelecimento dos pontos de corte para o número de repetições no teste de sentar e levantar de 30 segundos na predição da incapacidade na realização de ABVD e AIVD.

\section{Resultados}

Os 622 idosos avaliados possuíam média de idade de 71,08 anos $(\mathrm{dp}=7,77)$ com amplitude de 60 a 96 anos. Destes, $65 \%(n=404)$ eram do sexo feminino e $47,9 \%(n=298)$ encontravam na faixa etária de 60 a 69 anos.

A maioria dos idosos era de casados e/ou residentes com o parceiro $(57,2 \% ; n=357)$, tendo as mulheres a maior prevalência de viúvas. Havia maior frequência de mulheres idosas residentes em domicílios multigeracionais $(56,6 \%$; $\mathrm{n}=230)$. Quanto à escolaridade, $19,1 \%(\mathrm{n}=119)$ eram analfabetos, e os homens com a maior renda mensal (Tabela 1).

Com relação aos indicadores de saúde, as mulheres apresentaram piores resultados. Na saúde subjetiva $57,7 \%$ $(n=360)$ dos idosos reportaram o estado de saúde como negativa. A presença de déficit cognitivo foi identificada em $16,7 \%(\mathrm{n}=104)$ dos idosos. A maioria dos idosos relatou ter alguma doença $(95,4 \% ; n=595)$, o consumo de medicamentos foi descrito por $84,3 \%(n=526), 14,7 \%(n=92)$ foram hospitalizados nos últimos seis meses do período de entrevista e $29,4 \%(n=183)$ referiram ter sofrido queda no último ano (Tabela 1).

As tabelas 2 e 3 descrevem a comparação entre níveis de funcionalidade nas ABVD e AIVD, respectivamente, em relação às variáveis antropométricas e de desempenho físico (número de repetições no teste de sentar e levantar da cadeira durante 30 segundos).

Os resultados indicam não haver diferenças nas variáveis antropométricas quando comparado os grupos por 
Tabela 1. Distribuição das variáveis sociodemográficas e indicadores de saúde em relação ao sexo, Uberaba, MG, Brasil, 2010.

\begin{tabular}{|c|c|c|c|c|c|c|c|}
\hline \multirow[b]{2}{*}{ Variável } & \multicolumn{2}{|c|}{ Geral } & \multicolumn{2}{|c|}{ Homens } & \multicolumn{2}{|c|}{ Mulheres } & \multirow[b]{2}{*}{$p^{\mathrm{a}}$} \\
\hline & $\%$ & $\mathbf{n}$ & $\%$ & $\mathbf{n}$ & $\%$ & $\mathbf{n}$ & \\
\hline \multicolumn{8}{|l|}{ Faixa etária } \\
\hline 60-69 anos & 47,8 & 298 & 45,4 & 99 & 49,0 & 199 & 0,659 \\
\hline 70-79 anos & 36,5 & 228 & 37,6 & 82 & 36,0 & 146 & \\
\hline$\geq 80$ anos & 15,7 & 98 & 17,0 & 37 & 15,0 & 61 & \\
\hline \multicolumn{8}{|l|}{ Estado civil } \\
\hline Solteiro/separado/divorciado & 15,1 & 94 & 11,0 & 24 & 17,2 & 70 & 0,000 \\
\hline Casado/vivendo com parceiro & 57,2 & 357 & 75,2 & 164 & 47,5 & 193 & \\
\hline Viúvo & 27,7 & 173 & 13,8 & 30 & 35,2 & 143 & \\
\hline \multicolumn{8}{|l|}{ Arranjo domiciliar } \\
\hline Mora só & 11,9 & 74 & 10,1 & 22 & 12,8 & 52 & 0,001 \\
\hline Só o cônjuge & 25,6 & 160 & 33,0 & 72 & 21,7 & 88 & \\
\hline +filhos & 30,6 & 191 & 33,9 & 74 & 28,8 & 117 & \\
\hline+ netos & 24,2 & 151 & 17,4 & 38 & 27,8 & 113 & \\
\hline Outros & 7,7 & 48 & 5,5 & 12 & 8,9 & 36 & \\
\hline \multicolumn{8}{|l|}{ Anos de estudo } \\
\hline Analfabeto & 19,1 & 119 & 17,9 & 39 & 19,7 & 80 & 0,622 \\
\hline 1 a 2 anos & 21,2 & 132 & 19,7 & 43 & 21,9 & 89 & \\
\hline$\geq 3$ anos & 59,8 & 373 & 62,4 & 136 & 58,4 & 237 & \\
\hline \multicolumn{8}{|l|}{ Renda familiar } \\
\hline$\leq 1$ salário & 17,4 & 107 & 11,2 & 24 & 20,7 & 83 & 0,004 \\
\hline 1,1 a 2 salários & 34,1 & 210 & 38,6 & 83 & 31,7 & 127 & \\
\hline 2,1 a 4 salários & 33,9 & 209 & 31,6 & 68 & 35,2 & 141 & \\
\hline$\geq 4,1$ salários & 14,6 & 90 & 18,6 & 40 & 12,5 & 50 & \\
\hline \multicolumn{8}{|l|}{ Percepção de saúde } \\
\hline Positiva & 42,3 & 264 & 49,5 & 108 & 38,4 & 156 & 0,007 \\
\hline Negativa & 57,7 & 360 & 50,5 & 110 & 61,6 & 250 & \\
\hline \multicolumn{8}{|l|}{ Déficit cognitivo } \\
\hline Ausência & 83,3 & 518 & 85,3 & 186 & 82,2 & 332 & 0,36 \\
\hline Presença & 16,7 & 104 & 14,7 & 32 & 17,8 & 72 & \\
\hline \multicolumn{8}{|l|}{$\begin{array}{l}\text { Presença de doenças } \\
\text { autorreferidas }\end{array}$} \\
\hline Não & 4,6 & 29 & 7,8 & 17 & 3,0 & 12 & 0,006 \\
\hline Sim & 95,4 & 595 & 92,2 & 201 & 97,0 & 394 & \\
\hline \multicolumn{8}{|l|}{ Uso de medicamentos } \\
\hline Não & 15,7 & 98 & 22,9 & 50 & 11,8 & 48 & 0,000 \\
\hline Sim & 84,3 & 526 & 77,1 & 168 & 88,2 & 358 & \\
\hline \multicolumn{8}{|l|}{ Hospitalização } \\
\hline Não & 85,3 & 532 & 86,2 & 188 & 84,7 & 344 & 0,612 \\
\hline Sim & 14,7 & 92 & 13,8 & 30 & 15,3 & 62 & \\
\hline \multicolumn{8}{|l|}{ Quedas } \\
\hline Não & 70,4 & 439 & 75,7 & 165 & 67,5 & 274 & 0,032 \\
\hline Sim & 29,6 & 185 & 24,3 & 53 & 32,5 & 132 & \\
\hline \multicolumn{8}{|l|}{ ABVD } \\
\hline Independentes & 82,3 & 512 & 84,9 & 185 & 80,9 & 327 & 0,228 \\
\hline Dependentes & 17,6 & 110 & 15,1 & 33 & 19,1 & 77 & \\
\hline \multicolumn{8}{|l|}{ AIVD } \\
\hline Independentes & 270 & 43,4 & 36,2 & 79 & 47,3 & 191 & 0,009 \\
\hline Dependentes & 352 & 56,6 & 63,8 & 139 & 52,7 & 213 & \\
\hline
\end{tabular}

${ }^{\mathrm{a}}$ Qui-quadrado $\left(\chi^{2}\right)$ 
Tabela 2. Distribuição das variáveis antropométricas e número de repetição no teste de sentar em levantar em relação a condição de dependência para realização de atividades básicas da vida diária, Uberaba, MG, Brasil, 2010.

\begin{tabular}{lccccc}
\hline \multirow{2}{*}{ Variáveis } & \multicolumn{2}{c}{ Independente } & \multicolumn{2}{c}{ Dependente } & \multirow{2}{*}{$\boldsymbol{p}^{*}$} \\
\cline { 2 - 5 } & Média & dp & Média & dp & \\
\hline Antropometria & & & & & \\
Massa corporal $(\mathrm{Kg})$ & 67,83 & 14,35 & 69,55 & 17,24 & 0,332 \\
Estatura (centímetros) & 157,63 & 8,38 & 156,11 & 10,13 & 0,144 \\
Teste de sentar e levantar & & & & & \\
Número de repetições (média) & 12,07 & 4,47 & 7,79 & 5,27 & $\mathbf{0 , 0 0 0}$ \\
\hline * teste $t$ de Student para amostras independentes & & & &
\end{tabular}

Tabela 3. Distribuição das variáveis antropométricas e número de repetição no teste de sentar em levantar em relação a condição de dependência para realização de atividades instrumentais da vida diária, Uberaba, MG, Brasil, 2010.

\begin{tabular}{lccccc}
\hline \multirow{2}{*}{ Variáveis } & \multicolumn{2}{c}{ Independente } & \multicolumn{2}{c}{ Dependente } & \multirow{2}{*}{$\boldsymbol{p}^{*}$} \\
\cline { 2 - 5 } & Média & $\mathbf{d p}$ & Média & $\mathbf{d p}$ & \\
\hline Antropometria & & & & & \\
Massa corporal $(\mathrm{Kg})$ & 68,58 & 14,32 & 67,78 & 15,34 & 0,505 \\
Estatura (centímetros) & 157,35 & 7,98 & 157,37 & 9,27 & 0,971 \\
Teste de sentar e levantar & & & & & \\
Número de repetições (média) & 13,22 & 4,01 & 9,85 & 5,01 & $\mathbf{0 , 0 0 0}$ \\
\hline * teste $t$ de Student para amostras independentes & & & &
\end{tabular}

funcionalidade (dependente versus independente). Entretanto, para o teste de sentar e levantar houve diferenças entre os níveis de funcionalidade tanto para ABVD como para AIVD. A maior média do número de repetições no referido teste foi observado para o grupo que não possuía incapacidade funcional.

O teste de sentar e levantar mostrou-se como discriminador da incapacidade funcional devido aos tamanhos sob as áreas das curvas ROC serem superiores a 0,60 tanto para as ABVD como nas AIVD.

As Figuras 1 e 2 apresentam o ponto de corte com respectivas sensibilidades e especificidades do número de repetições do teste como preditor de incapacidade funcional nas ABVD e nas AIVD para ambos os sexos.

$O$ ponto de corte para determinar a incapacidade funcional nas ABVD para homens foi $\leq 10$ repetições e para as mulheres de $\leq 9$ repetições e nas AIVD de $\leq 14$ repetições para os homens e $\leq 10$ repetições para as mulheres (Figuras 1 e 2).

\section{Discussão}

A capacidade funcional engloba todas as funções do corpo, indica as interações positivas entre os indivíduos e os fatores do contexto ambiental e pessoal (Alves, Leite, \& Machado, 2008). Entretanto, a incapacidade está relacionada com os aspectos negativos desta interação, sendo idealizada como uma interação dinâmica entre os estados de saúde (doenças, perturbações, lesões, traumas, etc.) e os fatores contextuais (OMS, 2004).

A condição de incapacidade funcional eleva as dificuldades nas realizações das atividades típicas e pessoalmente desejadas na sociedade devido a uma deficiência (WHO, 1981). A incapacidade funcional encontrase em evidência nas políticas públicas relacionadas às condições de saúde da população idosa, pois a preservação da funcionalidade está atrelada a adoção de estilos de vida saudáveis (Virtuoso, Mendes, \& Tribess, 2010).

Considerando a etiologia da incapacidade funcional, em que as dificuldades em desempenhos físicos antecedem a instalação de processos incapacitantes, o conhecimento das limitações funcionais é útil para a identificação de pessoas mais vulneráveis a perda da funcionalidade. A identificação da força de membros inferiores tem se mostrado uma ferramenta interessante com bom potencial discriminador para fatores adversos à saúde, a exemplo de hospitalizações, quedas e fraturas (Kim, Yabushita, Kim, Matsuo, Okuno, \& Tanaka, 2010).

No presente estudo foi observado que mais da metade dos idosos são dependentes nas AIVD, enquanto nas ABVD a dependência foi observada em aproximadamente 1/4. Estes dados são similares a realidade de outros estudos brasileiros (Virtuoso \& Guerra, 2011; Maciel \& Guerra, 2007), sul (Del Duca, Da Silva, \& Hallal, 2009) e sudeste (Reyes-Ortiz, 2006), no qual constataram que mais da metade dos idosos eram totalmente independente para a realização das atividades básicas da vida diária.

A variedade de instrumentos utilizados para mensurar a capacidade funcional, assim como, os diferentes pontos de corte adotados para classificar a dependência funcional dificulta a comparação de resultados referentes as atividades básicas e instrumentais da vida diária (Paixão \& Reichenheim, 2005). Entretanto, as variáveis balizadoras da 

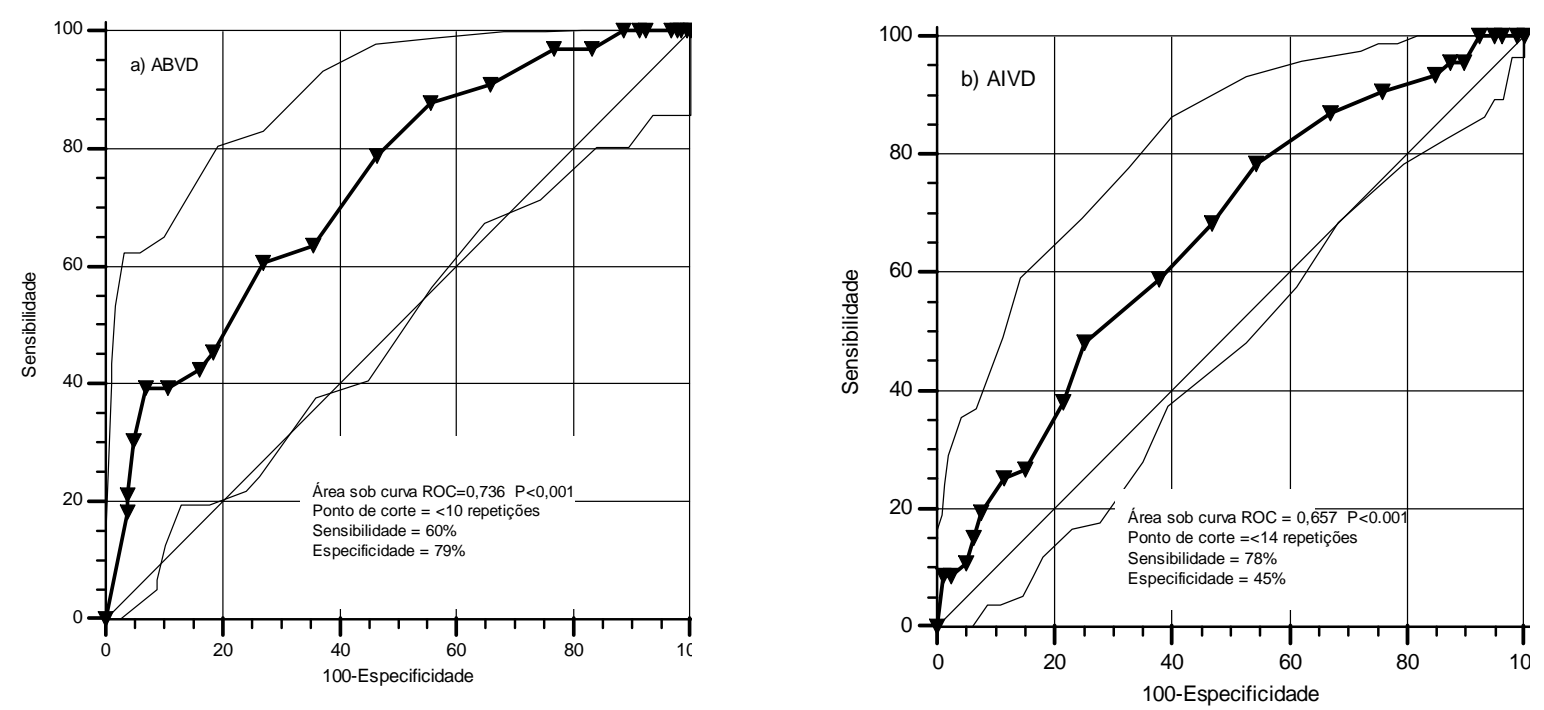

Figura 1. Área sob a curva ROC com o ponto de corte para o número de repetições no teste de sentar e levantar da cadeira como preditor para incapacidade funcional em homens idosos.
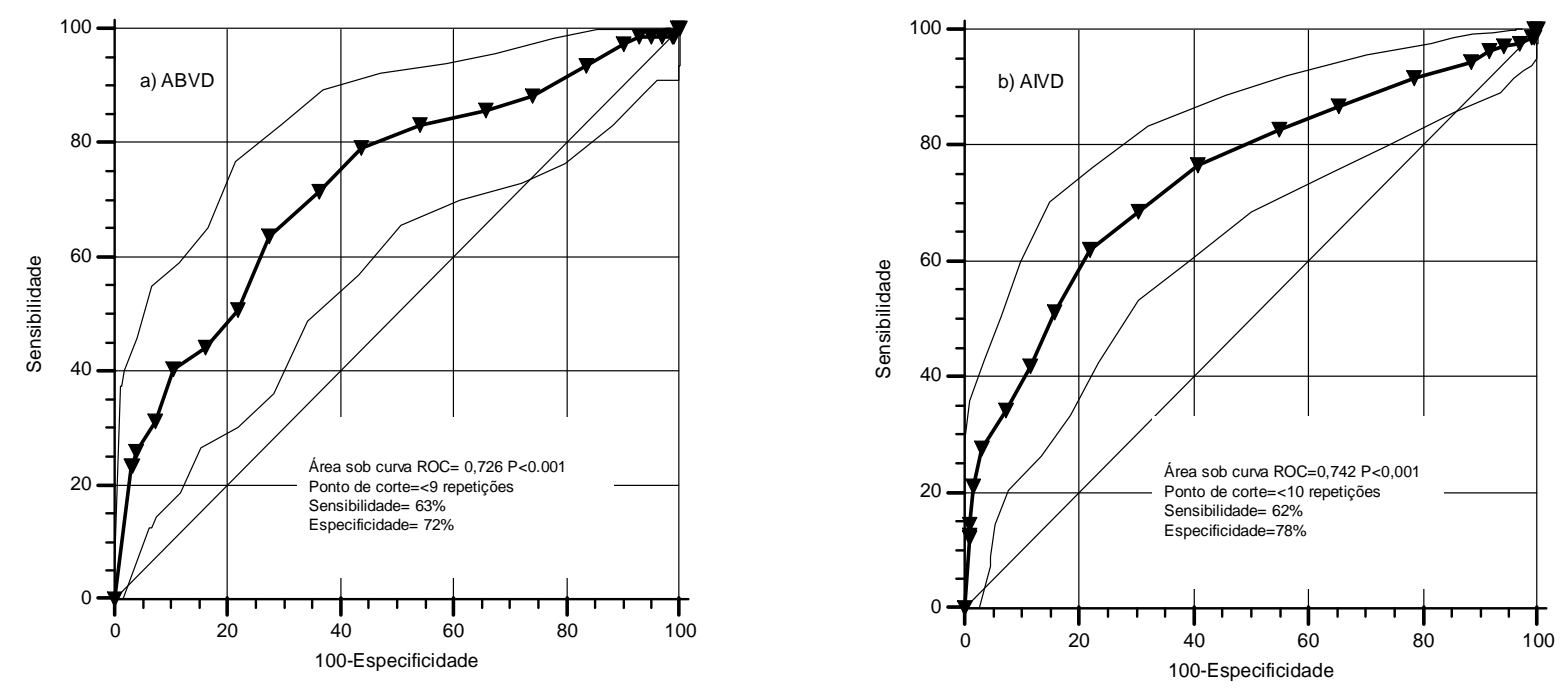

Figura 2. Área sob a curva ROC com o ponto de corte para o número de repetições no teste de sentar e levantar da cadeira como preditor para incapacidade funcional em mulheres idosas.

incapacidade funcional são semelhantes entre os estudos. Em geral, a prevalência de quedas em idosos dependentes nas ABVD é superior a aqueles idosos que não sofreram quedas. A ocorrência de quedas está diretamente associada com a diminuição da força muscular com o avançar da idade estando fortemente correlacionada com o desempenho na realização das AVD (Perracini \& Ramos, 2002; Puthoff \& Nielsen, 2007).

O envelhecimento aumenta o enfraquecimento muscular, caracterizada pela atrofia muscular das fibras tipo II, essa condição afeta a coordenação muscular, resultando em uma incapacidade funcional (Puthoff \& Nielsen, 2007) e muitas vezes ocasionando a mortalidade (Brito, Ricardo, Araujo,
Ramos, Myers, \& Araujo, 2012).

Os resultados desse estudo confirmaram que a força de membros inferiores pode ser utilizada como preditora da incapacidade funcional em idosos. Em estudo com amostra de 306 idosos residentes em comunidade japonesa foi identificado que tanto o teste de equilíbrio como de força de membros inferiores podem ser utilizados para identificação e monitoramento dos níveis hierárquicos de funcionalidade (Kim et al., 2010).

Partindo do pressuposto de que a tarefa de sentar e levantar do chão são básicos para a autonomia do idoso, Brito et al. (2012) realizaram estudo de coorte e identificaram que aqueles indivíduos com o menor escore no teste de sentar 
e levantar do chão, após ajuste por idade, sexo e índice de massa corporal obtiveram maiores riscos de mortalidade.

A habilidade de sentar e levantar da cadeira no período de 30 segundos envolvem mecanismos da função muscular e fatores complexos relacionados aos aspectos comportamentais (Cawthon et al., 2009; Jones, Rikli, \& Beam, 1999). Além desses aspectos, a redução da área muscular da coxa e/ou a redução da força muscular de membros inferiores estão relacionados à imobilidade e a redução de uma vida social ativa (Vaillancourt \& Newell, 2002).

$\mathrm{Na}$ análise do critério estabelecido para a força de membros inferiores como discriminador da incapacidade funcional foram observadas semelhanças entre os sexos nas ABVD. Entretanto, nas AIVD, o ponto de corte mais elevado foi determinado para os homens. Tais diferenças nas AIVD podem ser explicadas pelo padrão diferenciado no declínio da massa livre de gordura entre os sexos (Matsudo, Matsudo, \& Barros Neto, 2000). Nas ABVD, a condição de maior vulnerabilidade de eventos adversos à saúde limita a identificação de diferenças entre os sexos.

As medidas do declínio de força e potência muscular podem ser utilizadas como sinalizadores da incapacidade funcional (Brito et al., 2012; Puthoff \& Nielsen, 2007). Por outro lado, programas de treinamento de potência e força muscular em idosos proporcionam rápida recuperação na mobilidade e desempenho das tarefas diárias (Manini \& Clark, 2010).

O treinamento de força associado a outros tipos de treinamento, como o de flexibilidade e aeróbico, já tem sido recomendado pela comunidade científica, pois os mesmos trazem excelentes benefícios à saúde dos longevos com vistas a retardar os problemas advindos dos processos incapacitantes do envelhecimento (ACSM, 2011; Puthoff \& Nielsen, 2007).

O delineamento de corte transversal é uma limitação do estudo, pois impede o estabelecimento de causa e efeito entre as variáveis. No entanto, se trata de uma amostra representativa de idosos vinculados às equipes de saúde da família da localidade do estudo, minimizando a possibilidade do viés. Além disso, investigações com delineamento transversal são importantes, à medida que respaldam o desenvolvimento de ações de saúde por fornecerem dados imediatos da realidade do grupo observado e a geração de novas hipóteses.

Conclui-se que a força de membros inferiores, por meio teste de sentar e levantar da cadeira possui potencial de predição para a incapacidade funcional em pessoas idosas. Os escores iguais ou inferiores a 10 e 14 repetições (homens) e de 9 e 10 repetições (mulheres) possibilita a discriminação da incapacidade funcional, nas ABVD e AIVD, respectivamente.

\section{Referências}

Almeida, O. P. (1998). Mini exame do estado mental e o diagnóstico de demência no Brasil. Arquivos de Neuro-Psiquiatria, 56(3B), 605-612.
Alves, L.C., Leite, I. C., \& Machado, C.J. (2008). The concept and measurement of functional disability in the elderly population: A literature review. Ciência e Saúde Coletiva, 13(4), 1199-207.

American College Of Sports Medicine [ACSM]. (2011). Quantity and Quality of Exercise for Developing and Maintaining Cardiorespiratory, Musculoskeletal, and Neuromotor Fitness in Apparently Healthy Adults: Guidance for Prescribing Exercise. Medicine \& Science in Sports \& Exercise, 1334-1359.

Arai, Y. \& Hirose, N. (2013). Aging and Bio-motor function. Functional status, morbidity, and mortality of centenarians. Clinical Calcium, 23(1), 45-50.

Barreto, S. M., Pinheiro, A. R. O., Sichieri, R., Monteiro, C. A., Batista, M. Filho, Schimidt, M. I., ... Passos, V. M. A. (2005). Análise da Estratégia Global para Alimentação, Atividade Física e Saúde, da Organização Mundial da Saúde. Epidemiologia e Serviços de Saúde, 14(1), 41-68.

Brito, L. B. B.,Ricardo, D. R., Araujo, D. S. M. S., Ramos, P. S., Myers, J., \& Araujo, C. G. S. (2012). Ability to sit and rise from the floor as a predictor of all-cause mortality. European Journal of Preventive Cardiology 0(00), 1-7.

Cawthon, P. M., Fox, K. M., Gandra, S. R., Delmonico, M. J., Chiou, C. F., Anthony, M. S., Sewall, A., Goodpaster, B., Satterfield, S., Cummings, S. R., Harris, T. B.(2009). Do muscle mass, muscle density, strength, and physical function similarly influence risk of hospitalization in older adults? Journal of American Geriatric Society, 57(8), 1411-1419.

Chien, M.Y., Kuo, H. K., \& Wu, Y.T. (2010). Sarcopenia, cardiopulmonary fitness, and physical disability in communitydwelling elderly people. Physical Therapy, 90(9), 1277-87.

Del Duca G. F., Da Silva, M. C., Hallal, P. C. (2009). Incapacidade funcional para atividades básicas e instrumentais da vida diária. Revista de Saúde Pública 43(5), 796-805.

Ezzati, M., Friedman, A.B., Kulkarni, S.C., \& Murray, C.J. (2008). The reversal of fortunes: trends in county mortality and crosscounty mortality disparities in the United States. PLOS Medicine, 5(4), 557-568.

Folstein, M. F., Folstein, S. E., McHugh, P. R. (1975). "Minimental state" A practical method for grading the cognitive state of patients for the clinician. Journal of psychiatric research, 12(3), 189-198.

Instituto Brasileiro de Geografia e Estatística (2010). Censo demográfico 2010: resultados preliminares do universo. Recuperado em setembro, 2012, de: http://www.ibge. gov.br/ home/presidencia/noticias/noticia_visualiza.p hp?id_noticia $=1866 \& i d \_p a g i n a=1$

Instituto Brasileiro de Geografia e Estatística (2012). IBGE Cidades@, Minas Gerais. Recuperado em setembro, 2012, de: http://www.ibge.gov.br/cidadesat/link.php?cod mun=317010

Jones, C. J., Rikli, R. E., Beam, W.C. (1999). A 30-s chair-stand test as a measure of lower body strength in community-residing older adults. Research Quarterly for Exercise \& Sport, 70(2):113-9.

Kim, M., Yabushita, N., Kim, M., Matsuo, T., Okuno, J. \& Tanaka, K. (2010). Alternative items for identifying hierarchical levels of physical disability by using physical performance tests in women aged 75 years and older. Geriatrics \& Gerontology International, 10, 302-310.

Lino, V. T. S., Pereira, S. R. M., Camacho, L. A. B., Ribeiro, S. T. Filho., Buksman, S.(2008). Adaptação transcultural da Escala de Independência em Atividades da Vida Diária (Escala de Katz). Caderno de Saúde Pública, 24(1), 103-112.

Lohman, T. G., Roche, A. F., Martorell, R. (1998). Anthro-pometrics 
Standardization Reference Manual. Illinois: Human Kinetics Book.

Luiz, R. R., \& Magnanini, M. F. (2000). A lógica da determinação do tamanho da amostra em investigações epidemiológicas. Caderno de Saúde Coletiva, 8, 9-28.

Maciel, A. C. C., Guerra, R. O. (2011). Influência dos fatores biopsicossociais sobre a capacidade funcional de idosos residentes no nordeste do Brasil. Revista Brasileira de Epidemiologia, 10(2), 178-189.

Maciel, M. G. (2010). Atividade física e funcionalidade do idoso. Motriz, 16(4), 1024-1032.

Manini, T. M., \& Clark, B. C. (2012). Special Issue on Muscle Function and Sarcopenia. Dynapenia and Aging: An Update. Journal of Gerontolog A Biology Science and Medicine Science, 67A(1), 28-40.

Matsudo, S. M., Matsudo, V. K. R., de Barros Neto, T. L. (2000). Impacto do envelhecimento nas variáveis antropométricas, neuromotoras e metabólicas da aptidão Física. Revista Brasileira de Ciência do Movimento,8(4), 21-32.

McGough, E. L., Logsdon, R. G., Kelly, V.E., Teri, L. (2013). Functional mobility limitations and falls in assisted living residents with dementia: physical performance assessment and quantitative gait analysis. Journal of Geriatric Physical Therapy, 36(2), 78-86.

Nagi, S. Z. (1991). Model for disability and disability prevention. Pope, A. M., Taylor, A. R., (Eds). Disability in America: toward a national agenda for prevetion (pp 76-104). Washington: National Academy Press.

Organização Mundial de Saúde. (2004). CIF: Classificação Internacional de Funcionalidade, Incapacidade e Saúde. Direção Geral da Saúde. Lisboa.

Paixão, C. M. Jr., Reichenheim, M. E. (2005). Uma revisão sobre instrumentos de avaliação do estado funcional do idoso. Cadernos de Saúde Pública, 21(1), 7-19.

Perracini, M. R., Ramos, L. R. (2002). Fatores associados a quedas em uma coorte de idosos residentes na comunidade. Revista de Saúde Pública,36(6), 709-16.

Puthoff, M.L., Nielsen, D. H. (2007).Relationships among impairments in lower-extremity strength and power, functional limitations, and disability in older adults. Physical Therapy, 87(10), 1334-47.

Reuben, D. B. \& Solomon, D.H. (1989). Assessment in geriatrics. Of caveats and names. Journal of American Geriatric Society, 37(6), 570-2.

Reyes-Ortiz, C. (2006). Cross-national comparison of disability in Latin American and Caribbean persons aged 75 and older. Archives of Gerontology and Geriatrics, Amsterdam, 42(1), 21-23.

Rikli, R. E., Jones, C. J. (1999). Development and validation of a functional fitness test for community-residing older adults. Journal of Aging and Physical Activity, 7, 129-61.

Rikli, R. E., Jones, C. J. (2013). Development and validation of criterion-referenced clinically relevant fitness standards for maintaining physical independence in later years. Gerontologist, 53(2), 255-67.

Rikli, R. E., Jones, C. J. Parâmetros do TAFI. In: Rikli, R. E., Jones, C. J. (Eds). Teste de aptidão física para idosos (Cap.2, pp.13-27). Barueri: Manole, 2008.

Roorda, L. D., Roebroeck, M. E., Lankhorst, G. J., Tilburg, T. Van., Bouter, L. M., (1996). Measuring Functional Limitations in Rising and Sitting Down: Development of a Questionnaire. Archives of Physical Medicine and Rehabilitation, 77, 663-9.

Santos, R. L., Virtuoso, J. S. Jr. (2008). Confiabilidade da versão brasileira da escala de atividades instrumentais da vida diária.
Revista Brasileira em Promoção da Saúde, 21(4), 290-296.

Vaillancourt, D. E., Newell, K. M. (2002). Changing complexity in human behavior and physiology through aging and disease. Neurobiology Aging, 23(1), 1-11.

Verbrugge, L. M., \& Jette, A. M. (1994). The disablement process. Social Science \& Medicine, 38(1), 1-14.

Virtuoso, J. S. Jr., Guerra, R. O. (2011). Incapacidade funcional em mulheres idosas de baixa renda. Ciência \& Saúde Coletiva, 16(5), 2541-2548.

Virtuoso, J. S., Jr., Mendes, E. L., Tribess, S. (2010). Envelhecimento, Saúde e Capacidade Funcional. In: Moreira, W. W. (Orgs.). Educação Física, esporte, saúde e educação (pp. 317-332). Uberaba- MG: Universidade Federal do Triângulo Mineiro.

Wennie, H.W. N., Perera, S., Swearingen, J. Van., Studenski, S. Performance measures predict onset of activity of daily living difficulty in community-dwelling older adults. J American Geriatric Society, 58(5), 844-52.

World Health Organization. (1981). Disability prevention and rehabilitation. Geneva.

Worral, L., McCooey, R., Davidson, B., Larkins, B., \& Hickson, L. (2002). The validity of functional assessments of communication and the activity/participation components of the ICDH-2: do they reflect what really happens in reallife? Journal of Communication Disorders, 35(2), 107-137.

\section{Nota dos autores}

Rafaela G. dos Santos, Joilson Meneguci, Letícia L. A. da G. Bastos. Mestrandos em Educação Física - Universidade Federal do Triângulo Mineiro. Uberaba, MG, Brasil; Núcleo de Estudos em Atividade Física e Saúde (NEAFISA); Bolsistas CAPES.

Renata Damião. Departamento de Nutrição e Programa de PósGraduação em Educação Física da Universidade Federal do Triângulo Mineiro. Uberaba, MG, Brasil; Núcleo de Estudos em Atividade Física e Saúde (NEAFISA).

Jair S. V. Júnior, Sheilla Tribess. Departamento de Ciências do Esporte e Programa de Pós-Graduação em Educação Física da Universidade Federal do Triângulo Mineiro. Uberaba, MG, Brasil; Núcleo de Estudos em Atividade Física e Saúde (NEAFISA).

\section{Correspondência}

\section{Jair S. V. Júnior}

Av. Tutunas, 570, Secretaria da Pós-Graduação em Educação Física. Bairro Tutunas, Uberaba, MG, 38061-500 Brasil

E-mail: jair@ef.uftm.edu.

Telefone: (34) 3318-5067

Artigo completo de trabalho apresentado no VIII Congresso Internacional de Educação Física e Motricidade Humana e XIV Simpósio Paulista de Educação Física.

Declaração de Conflito de Interesses: Os autores declararam ausência de conflitos de interesse no que diz respeito à pesquisa, autoria e / ou publicação deste artigo.

Manuscrito recebido em 30 de março de 2013

Manuscrito aceito em 10 de maio de 2013 